\title{
"Cooking and Active Leisure" TAS Program, Spain: A Program Impact Pathways (PIP) analysis
}

\author{
Elena Roura, Sara Lucía Pareja, Raimon Milà, and Núria Cinca
}

\begin{abstract}
Background. The "Cooking and Active Leisure" Tu y Alícia por la Salud (CAL-TAS) Program is a schoolbased pilot that addresses healthy lifestyle needs of Spanish secondary school students with initiatives that research has proven to improve dietary and physical activity behaviors.

Objective. The objectives were to perform a Program Impact Pathways (PIP) analysis to describe key activities and processes of the CAL-TAS Program, identify Critical Quality Control Points (CCPs), and identify a suite of common indicators of healthy lifestyles to be applied across participant schools.
\end{abstract}

Methods. The CAL-TAS Program designers and implementation team developed this PIP analysis through an iterative process and presented the results for feedback at the seven-country Healthy Lifestyles Program Evaluation Workshop held in Granada, Spain, 13-14 September 2013, under the auspices of the Mondelez International Foundation.

Results. The team identified three PIP CCPs: teachers' motivation and training, changes in students' knowledge of healthy lifestyles, and changes in students' healthy lifestyle behavior. The selected indicators of the program's impact on healthy lifestyles are adequacy of food intake, level of knowledge of healthy lifestyles gained,

The authors are affiliated with the Alícia Foundation, Barcelona, Spain; Raimon Milà is also affiliated with Vic University, Barcelona, Spain.

Please direct queries to the corresponding author: Elena Roura, Camí de St. Benet, s/n, 08272 Sant Fruitós del Bages, Barcelona, Spain; e-mail: elena@Alícia.cat.

This contribution forms part of the proceedings of the workshop "Identifying common metrics for evaluating school-based healthy lifestyles programs in seven countries," held in Granada, Spain, 13-14 September 2013. Guest editors: Rafael Pérez-Escamilla, Ph.D., Yale School of Public Health, New Haven, Connecticut, USA, and Michael Alberg-Seberich, M.A., Forum for Active Philanthropy, Berlin, Germany. This workshop was funded by the Mondelēz International Foundation. and adequacy of physical activity level according to World Health Organization recommendations. A clear definition of impact indicators, as well as collection of accurate data on healthy lifestyle behaviors and knowledge, is essential to understanding the effectiveness of this program before it can be scaled up.

Conclusions. CAL-TAS is an effective secondary school-based program encouraging healthy lifestyles. The PIP analysis was instrumental in identifying CCPs to sustain and improve the quality of the program. The team hopes to sustain and improve the program through these program evaluation recommendations.

Key words: Eating habits, food consumption, impact indicators, physical activity, program evaluation, Program Impact Pathways, school, Spain, teenagers

\section{Background}

In the European Union, approximately $50 \%$ of adults and more than $20 \%$ of children of school age (corresponding to 260 million adults and more than 12 million children) are overweight or obese [1]. Spain is classified by the World Health Organization as one of the EU countries with the highest prevalence of overweight and obese children [2,3]. According to the 2011 National Health Survey of Spain, 27.8\% of Spanish children between 2 and 17 years of age weigh more than recommended [4]. Nearly 3 in 10 children are overweight or obese, double the number compared with 20 years ago [5]. Since the late 1990s, the Spanish government has developed programs and strategies to tackle obesity, including the Ministry of Health Nutrition, Physical Activity, and Obesity (NAOS) prevention strategy established in 2005. Despite several public- and private-sector projects, the complexities of the obesity epidemic have prevented these efforts from being effective [6].

One of the most important causes of youth obesity is modern lifestyles with regard to eating and physical 
activity behaviors. This situation must be addressed primarily in childhood and adolescence; school-based healthy lifestyles programs are likely to be most effective in reaching these age groups. A large number of school programs have been implemented in recent years, most of them focused on young children and excluding adolescents [7]. This is a public health concern, as adolescence is one of the most dynamic and complex life transition phases. The physical, developmental, and social changes that occur during adolescence can markedly affect long-term eating and physical activity behaviors. Nutrient needs during adolescence are higher than at any other time, and an inadequate diet during adolescence can affect growth and development. Inappropriate diet may also increase the risk of a number of immediate health problems, including eating disorders and obesity, as well as the potential for chronic diseases. Growing independence and eating away from home, concern with physical appearance and body weight, the need for peer acceptance, and busy schedules all have an effect on eating patterns and food choices during adolescence and beyond. Therefore, adolescence offers a unique opportunity to positively influence the adoption of healthful eating and physical activity patterns that may be sustained throughout life [8].

Despite widespread awareness of the importance of healthy eating in adolescence, healthy eating messages are rarely put into practice, and nutritional problems are prevalent among Spanish youth. Many adolescents engage in unhealthy dieting practices, and many youth eating patterns include high intakes of fast foods and other foods high in sodium, sugar, and fat, alongside low intakes of fruits, vegetables, and calcium-rich foods. In studies to date, teens demonstrate a lack of concern for healthy eating and express little normative social support for developing healthy eating habits. Although healthcare professionals, educators, parents, and others encourage teens to "eat healthy," it is essential to understand how adolescents perceive healthy eating recommendations and their associated health benefits. This understanding can facilitate the development of effective interventions to create normative support for healthy eating among adolescents and assist them in adopting the recommendations [9].

School-based healthy eating and physical activity programs provide a great opportunity to improve the future health and well-being of children. Children and adolescents spend the majority of their day at school, and the school environment is recognized as having a powerful influence on their physical activity and eating behaviors $[10,11]$. Thus, schools can play a vital role in educating children about making healthy nutritional, physical, and psychological choices. Through schools, these lessons can reach almost all children and may enhance learning and provide social benefits, enhance health during critical periods of growth and maturation, lower the risk of chronic diseases in adulthood, and help to establish healthy behaviors at an early age to create lifelong healthy habits. The effectiveness of school-based healthy eating and physical activity programs is critical for evidence-based health policy and for justifying broader implementation of successful programs. However, only a limited number of studies have been conducted on these programs, and the results have varied. The effectiveness of these programs is not well established [12]. Implementing effective, evidence-based intervention programs to promote physical activity and healthy eating among adolescents means properly designing interventions from the outset to thoroughly assess their effectiveness in changing behavior.

With support from the Mondelēz International Foundation, the "Cooking and Active Leisure" Tu y Alícia por la Salud (CAL-TAS) Program was born in 2011, based on the idea that adolescence is a time to instill and sustain healthy lifestyles. CAL-TAS is designed as a healthy lifestyle promotion educational pilot program, with a strong evaluation component to assess its impact on dietary and physical activity behavioral changes.

The program is focused on teenagers because few programs have been specifically designed to target this age group. The CAL-TAS curriculum is built upon the premise that changing lifestyle habits among young people is not easy. With adolescents, it is not enough to simply provide information on health problems and how to prevent them; to improve lifestyles, teenagers themselves must play an active role in the program [8]. As demonstrated by initiatives undertaken by the Alícia Foundation, the best results in improving healthy habits have included activities proposed by students going beyond passive nutrition and health education. The following section describes the CAL-TAS Program in detail.

\section{Description of the CAL-TAS Program}

\section{Target audience and setting}

CAL-TAS (www.programatas.com) is a program to promote and improve eating and physical activity habits in students aged 14 and 15. It is delivered through 103 classes in 79 secondary schools located in all autonomous regions of Spain.

\section{Program goals}

The final goal of CAL-TAS is to improve eating habits and promote physical activity in teenagers through an interactive on-line tool. This public domain tool will allow any interested secondary schools to replicate the program to improve healthy lifestyles using a proven 
and effective methodology. The CAL-TAS Program's objectives are to generate a deep knowledge of the eating habits and physical activity of Spanish teenagers; demonstrate that cooking can be a good tool to encourage healthy eating and generate changes in teens' behavior; promote active leisure time as a vehicle to increase physical activity; and prove that active participation of teens in designing strategies to improve their habits is one of the most effective ways to modify them, as their own proposals are in concordance with their generation, lifestyles, and preferences.

Working toward these objectives, the implementation program was completed in two phases.

\section{Phase 1}

\section{Teacher training}

The program began with a training session for teachers to ensure correct program implementation. They were taught how to complete food records and physical activity questionnaires and how to transfer the data collected.

\section{Food and physical activity records}

Students, with teachers' help, kept track of their food and physical activity records using a Web-based portal.

\section{Diet and physical activity report}

The Alícia Foundation wrote a report for each school summarizing baseline food consumption and physical activity habits and highlighting areas that need improvement. Once the reports were reviewed by the teachers at each participating class, the program was initiated by implementing the program's healthy lifestyle educational curriculum.

\section{Healthy lifestyle educational curriculum}

The "CAL-TAS Route" curriculum consisted of two workshops. The first, "Looking for balance," addressed nutrition and physical activity principles, while the second, "I cook and it's mine to eat," used culinary skills as a strategy for healthier eating habits. After participation in the two workshops and based upon the school-specific healthy lifestyle baseline report highlighting nutrition and physical activity habits, the students worked together for 6 months to design a strategy to promote healthy eating and physical activity habits to implement in their own schools. Additional monthly challenges related to increasing the consumption of healthy foods or physical activity levels were also presented by the Alícia Foundation. In order to meet the challenge, students needed to plan and implement solutions. Throughout the entire period, both teachers and students were supported by a team from the Alícia Foundation. Once the student proposals were completed, each class sent its CAL-TAS proposal to the Alícia Foundation to be evaluated by an expert jury (pictures 1 and 2).

\section{Selection of schools to continue in the program}

The 10 schools with the best proposals were selected to continue the program in the next school year (phase 2).

\section{Phase 2}

The 10 selected schools implemented a weeklong series of "TAS workshops," which focused on the promotion of healthy eating and physical activity habits for the entire school, including students, teachers, and parents.

As in the first phase, activities were focused on cooking and physical activity during leisure time, but they

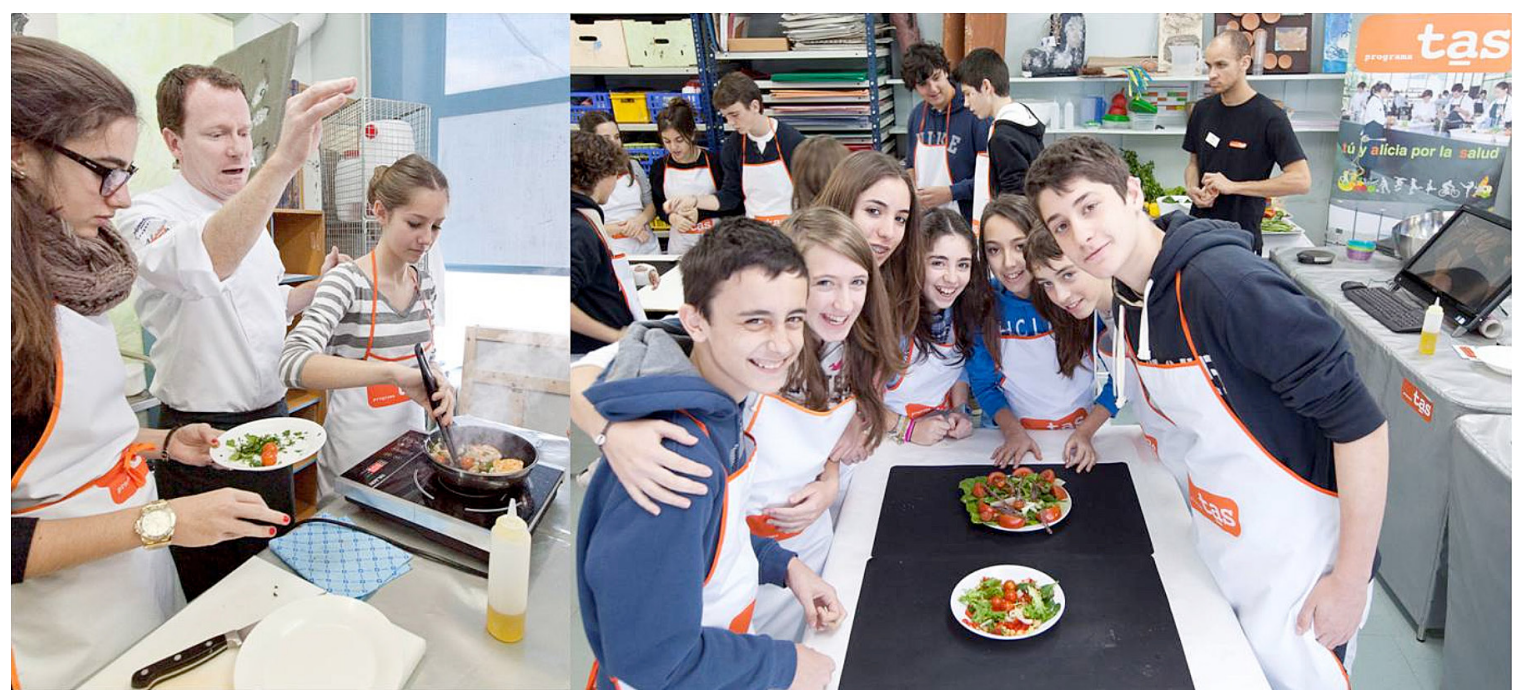

PICTURE 1. Cooking workshop "I cook and it's mine to eat" in El Puig School, Esparraguera, Barcelona, Spain. Courtesy of Fundación Alícia, Barcelona 


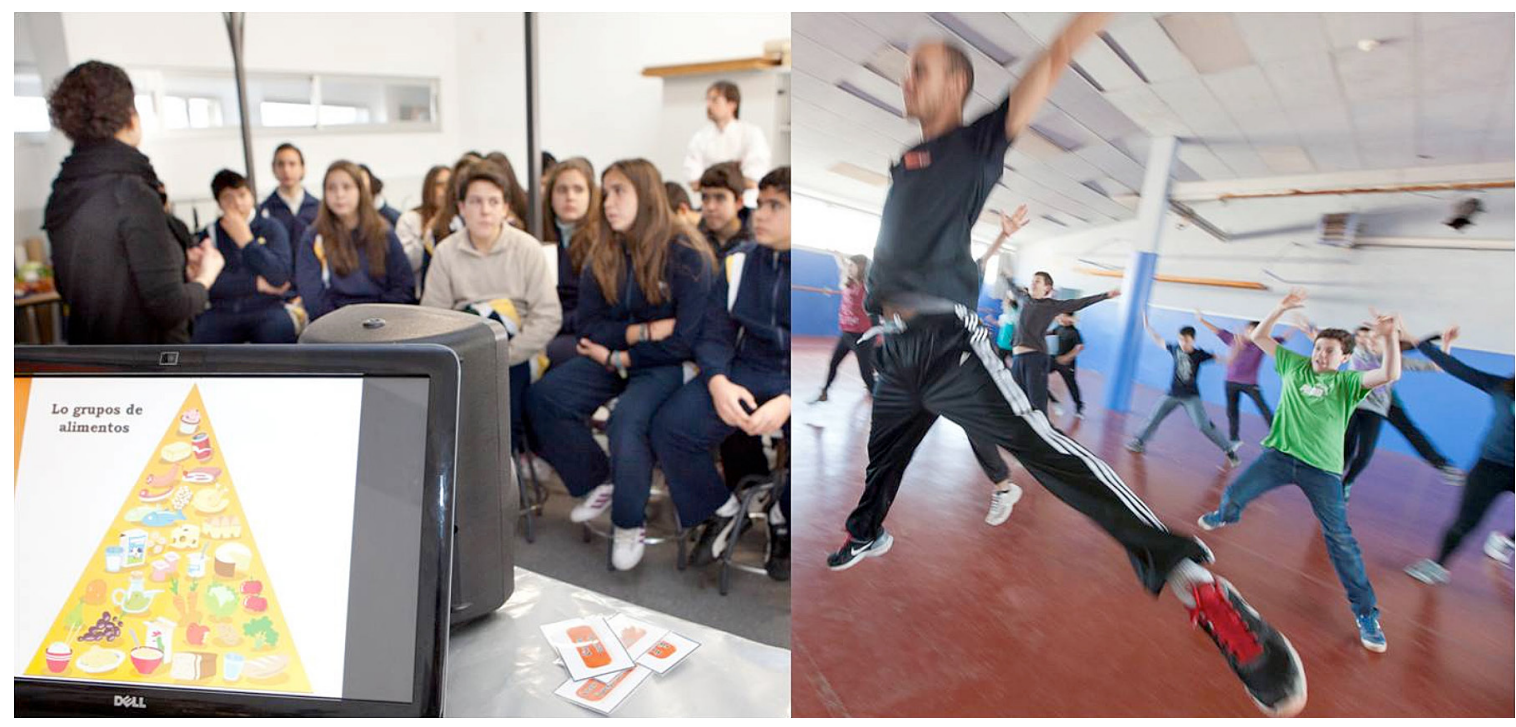

PICTURE 2. Activity "Looking for balance" in La Salle Felipe Benito de Sevilla School, Spain. Courtesy of Fundación Alícia, Barcelona

also included activities about food awareness and prevention of disordered eating behaviors. The food and cooking workshops included "What do people cook around the world?," "Eating well," "How to plan healthy eating!", and "I cook and it's mine to eat." The following interactive workshops were also added to increase physical activity: "Do I live an active life?," "The playground, a place to move around!," "Dance," and "Make the most of your surroundings to be active!" The food awareness and perception workshops included "Searching for balance," "I like myself just the way I am," and "The senses, the body windows!"

The team also developed workshops for adults who interact with youth. The "Need to feel well" workshop was designed to help teachers and parents detect and prevent eating disorders among adolescents. Teachers and parents were also offered the "Cooking food to eat better" and "Nothing gets wasted here" workshops, which taught practical and tasty ways of preparing healthy meals.

The project also uses an innovative methodology to involve teens as active agents of change. Students who participated in the development of their school's proposals during phase 1 were designated as proactive participants during these phase 2 activities. While implementing their winning proposals, the teens simultaneously developed their own strategies and tools to promote healthy lifestyles (focused around cooking and active leisure) to improve the habits of their class.

\section{Program evaluation: CAL-TAS Program, phase 1}

As indicated above, program evaluation was a critical component of CAL-TAS since its inception. Measuring the impact of the program on students is the only way to demonstrate the program's impact. The program evaluation was conducted by the Alícia Foundation with funding provided by the Mondelēz International Foundation. It assessed the food habits and physical activity of a sample of 2,516 students 14 and 15 years of age. The sample set included students from $103 \mathrm{sec}$ ondary school classes, representing public, private, and charter schools of all the autonomous Spanish regions. The evaluation was based on a pre- postprogram exposure design. Surveys were administered to assess food consumption habits, level of physical activity, and knowledge gained about healthy eating and being physically active at baseline and following the education intervention. The dietary intakes of the students were assessed from food records in which each participant had to report all foods and beverages consumed for a period of 1 week. For each food or beverage recorded, the student reported if it was whole fat, low fat, and/or sugar sweetened, how it was prepared (baking, frying, etc.), and recipes for mixed dishes. The students also reported portion sizes consumed. The students filled out the surveys and transmitted the data to the Alícia Foundation over a Web-based platform designed for this program.

The nine-item Physical Activity QuestionnaireAdolescents (PAQ-A) recorded self-reported physical activity during the previous 7 days. Students reported activities performed during leisure time, during physical activity classes, and at different times (lunch, afternoon, evening) throughout the week-long reporting period. Preliminary results demonstrate that the CAL-TAS Program is improving dietary and physical activity behaviors among teenagers. Results from 
the baseline assessment indicated very low compliance with nutritional and physical activity recommendations. These behaviors improved after a school cycle of program exposure (figs. 1 and 2). Specifically, the proportion of student participants who met the minimum recommendations for fruit and vegetable intake increased by $11 \%$ and $13.8 \%$, respectively $(p<.05)$. Legume consumption also significantly improved from a mean of 1.6 servings per week to 1.9 servings per week $(p<.05)$, with a $6 \%$ increase in the proportion of students who met the minimum recommendation for legume consumption. Significant improvement was also seen in fish intake, with an increase of almost $6 \%$ in the proportion of students who met the minimum recommendation for fish consumption $(p<.05)$.

Besides these improvements in consumption of nutritious foods, it is worth noting that there were substantial reductions in the consumption of sugarsweetened beverages (by 1.3 servings per week) and of sweet baked goods, snacks, cakes, and cookies (by two servings per week), compared with the baseline evaluation ( $p<.05$ for both changes). The level of physical activity also improved significantly (fig. 2). The proportion of students reporting an insufficient level of physical activity decreased from $35.6 \%$ at baseline to $30.7 \%$ after exposure to the intervention $(p<.05)$.

In summary, preliminary results indicate that the CAL-TAS Program has a positive impact on dietary and physical activity behaviors. This, however, raises the important question of how this impact is being achieved. This was the basis of our Program Impact Pathways (PIP) framework [13].

\section{Objective}

The objective of this study was to develop a PIP analysis to describe the key activities and processes of the CAL-TAS Program, identify program Critical Quality Control Points (CCPs), and identify a suite of common healthy lifestyle indicators that should be collected by all participant schools.

The Mondelēz International Foundation hosted a Healthy Lifestyles Program Evaluation Workshop in Granada, Spain, on 13-14 September 2013, with the goal of identifying common metrics among schoolbased healthy lifestyles programs supported by the foundation in seven countries. This was also an opportunity for the Alícia Foundation to present their initial PIP analysis for feedback from other workshop participants and the guest editors of this Supplement.

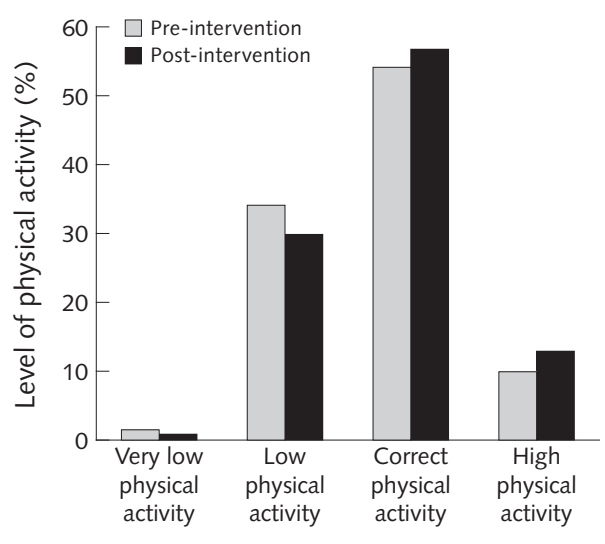

FIG. 2. Percentage of children performing different levels of physical activity, according to Physical Activity Questionnaire-Adolescents (PAQ-A), pre- and postintervention (phase 1)

\section{Methods}

The CAL-TAS Program PIP analysis was developed through an iterative process, with input from key staff, including the Alícia Foundation's Project Development Manager Dr. Elena Roura and project Field Director Sara Lucia Pareja, in partnership with program evaluation director Dr. Raimon Milà from Vic University. The PIP analysis process involved detailed reviews of the initial project proposal, the schools' annual program reports, and program operational and technical materials, as well as several round-table discussions with the program implementation team.

Following the Workshop Evaluation Manual developed in preparation for this workshop [15], the team described the program's logic model, including a narrative description of the goals, target audience and setting, inputs, activities, outputs, and preliminary evaluation findings. Then, through a series of meetings 
conducted with key program staff, the CAL-TAS PIP diagram was developed. This allowed us to identify the key program activities and processes linking these activities, the CCPs, a suite of SMART (Specific, Measurable, Achievable, Relevant, Timely) healthy lifestyle indicators to assess the expected program impact, and an evaluation design that could be used to improve the program's future impact assessments.

After performing the initial PIP analyses, the team submitted a first draft of the CAL-TAS PIP report for review by lead workshop evaluation expert Dr. Rafael Pérez-Escamilla before the Granada workshop. Finally, the feedback received at that workshop was incorporated into the findings presented here.

\section{Results}

The PIP analysis was used to understand the ways in which the CAL-TAS Program can achieve its objectives and identify the CCPs for improving the quality of the program. The PIP diagram (fig. 3) presents the architecture of the program. As shown in the PIP diagram, the following conditions must be met to gain access to target teenagers (ages 14 and 15), who benefit from the CAL-TAS Program intervention:

" Build a multidisciplinary group of professionals capable of generating innovative curriculum and workshops on healthy food, cooking, and physical activity. The team brought together a group of diet and nutrition specialists, sports scientists, and educational and adolescent pedagogical specialists.

» Motivate and incentivize the target schools to enroll in the program. A good communication strategy combined with local health agents participating as evaluators was key to enrolling schools. The selected schools also received a cash award of 200 euros as a modest monetary incentive.

" Generate interest among the teachers responsible for delivering the program workshops to students. Teachers were invited to training sessions at the Alícia Foundation in Barcelona, with expenses paid. The event also afforded them the opportunity to meet with teachers from across the country in professional and social settings. This proved to be a strong incentive for teachers, as secondary school teachers in Spain rarely have the opportunity to attend these kinds of activities.

The results of these PIP analyses helped us corroborate the great opportunity that the school and teachers have to positively influence teenagers when a schoolbased intervention program that receives strong input from the teens themselves is well designed and implemented. As a result, the program operations manual now includes the importance of ensuring that these engagement activities are closely monitored.

Once the three conditions detailed above have been met, students are exposed to the CAL-TAS Program and become aware of and participate in its different activities. The diagram shows that the students must learn from the program activities (CCP 1) in order to change their behaviors related to healthy dietary and physical activity patterns (CCP 2).

The PIP analysis demonstrated that for the CAL-TAS Program to operate efficiently and improve students' lifestyles, the program must provide schools, teachers, and students with results of the baseline evaluation specific to their classrooms. The PIP analysis indicates that a key next step is for the CAL-TAS curriculum to directly and actively involve the students in making the necessary changes in their lifestyles, using their own proposed strategies to improve diet and physical activity. To achieve this, the CAL-TAS curriculum is flexible, taking into account the students' preferences, lifestyles, and technology requirements. Following the PIP analysis, the team is now considering providing additional incentives to increase the teens' motivation and encourage them to participate in all the program activities as active agents of change.

Phase 1 of the CAL-TAS Program does not address issues related to access to healthy foods and opportunities for physical activity in their homes or neighborhoods. However, the PIP analysis strongly suggests that even in the absence of activities to facilitate access to healthy foods and opportunities for physical activity, it is reasonable to expect that CAL-TAS by itself can lead to improvements in healthy lifestyles. This assumption is based on the fact that teenagers are very independent when it comes to what they eat and what they do in their free time. We acknowledge, however, that this key assumption may not be realistic for younger children. The PIP analyses also led the team to conclude that future activities should be strengthened by understanding and addressing the family and whole-school environments.

CAL-TAS is a pilot intervention program. For this reason, it is essential to continue systematically evaluating its impact as it evolves. In order to understand whether the CAL-TAS Program is meeting its stated goals, different impact indicators are measured, including changes in dietary habits and physical activity levels among participating students through weekly registers before and after the two program phases described above, and changes in students' knowledge of dietary and physical activity recommendations through prepost evaluations in the second phase. As shown in the PIP diagram, three CCPs are based on pre-post behavior evaluations in phases 1 and 2 of the program, and two CCPs are based on pre-post knowledge evaluations in phase 2. The PIP diagram also highlights the importance of tracking change in knowledge of healthy lifestyles as the program continues to evolve. The PIP analysis also calls for assessing teachers' satisfaction with the CAL-TAS Program. Both PIP-informed 


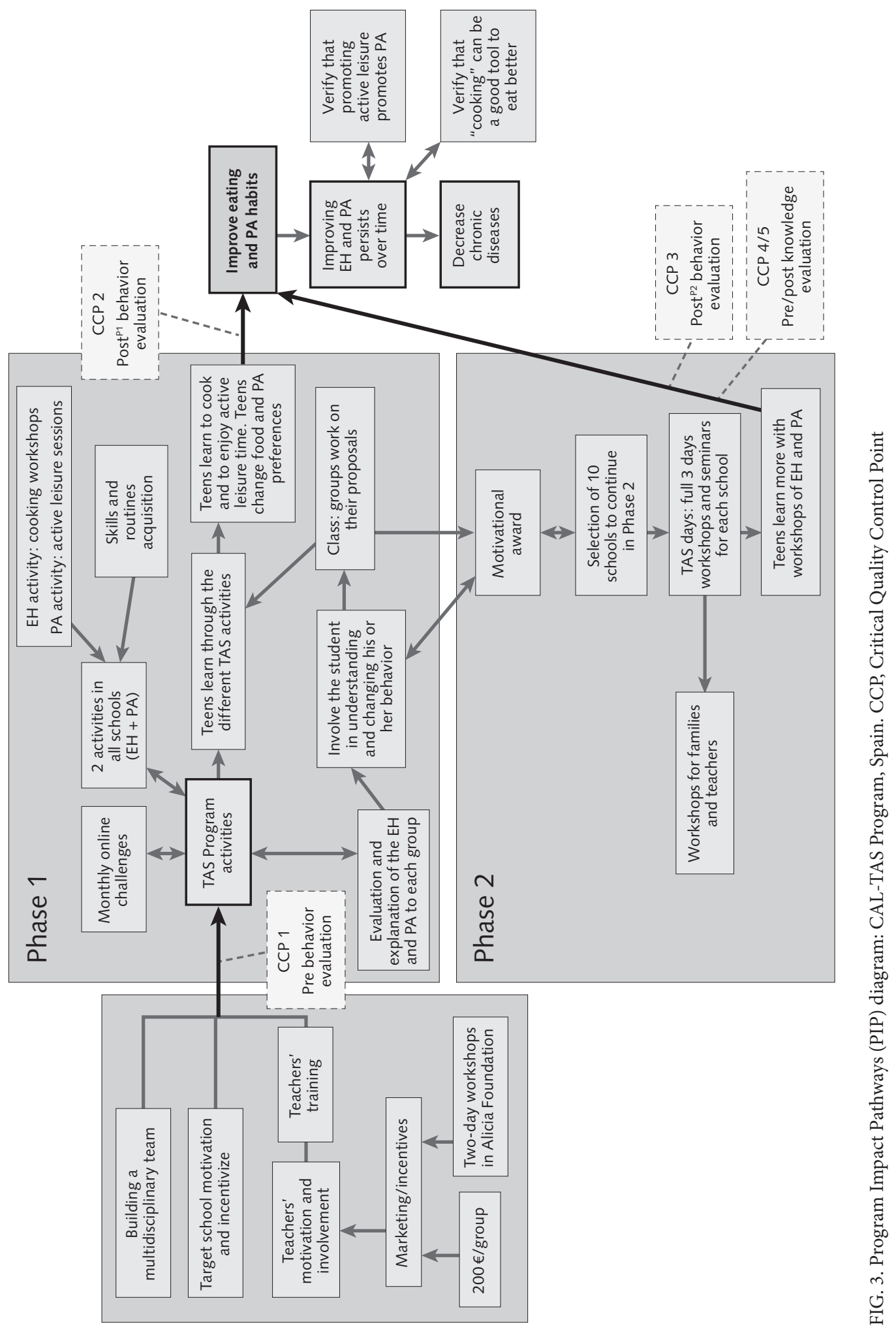


evaluation recommendations are currently being implemented.

As described before, the evaluation of phase 1 of the program showed significant improvements in eating habits and physical activity after exposure to the program. Nonetheless, the PIP analysis made us realize that it is important to acknowledge that there may be other activities unrelated to our program that could also explain these improvements.

As a result of the discussions of PIP analyses, a new data quality control system has been developed that will be implemented by the Alícia Foundation professionals with the following additions: validation of students' food consumption notebooks, standardization of food rations to ensure valid reporting of portion size by students, review of food records by the teachers responsible for each group before they are sent to the Alícia Foundation, and addition of plausible value ranges in the Web-based data entry platform to reduce inaccurate reporting of food consumption and physical activity data by students.

Although CAL-TAS does want to contribute to the prevention of chronic diseases, the PIP analyses led us to conclude that we cannot include this as a primary outcome of the program, as there are no plans to track this goal.

\section{Discussion}

In this study, the CAL-TAS team performed a PIP analysis to evaluate the implementation, coverage, and effectiveness of a school-based pilot program intervention to promote healthy lifestyles among adolescents. The program activities are based on cooking workshops, active leisure sessions, and activities designed by the target secondary school students themselves. The process of developing the PIP analysis was very useful for the implementation and evaluation staff, as it helped the team to collectively clarify program aims, develop realistic expectations regarding the impact of program activities, identify the CCPs to be monitored as CAL-TAS moves into the next phase of the program, and identify achievable impact indicators. The PIP analysis has been a useful tool as the program looks to move beyond the pilot stage into delivering curriculum to the schools and monitoring quality and impact via a Web-based platform.

One of the primary benefits of the PIP analysis was the ability to clearly identify and differentiate the program aims to promote active participation of teens in designing strategies to improve their lifestyle habits, promote cooking as a tool to encourage healthy eating and to generate sustainable changes in teens' dietary behaviors, and promote active leisure as a vehicle to increase physical activity. This process has helped the project implementation and evaluation team to better understand the path CAL-TAS must to take to achieve its overall desired impact as it expands and refines its activities in the third phase of the program.

The project is currently in phase 3 (begun in September 2013), and there is still much work to be done and many critical points to be resolved, such as the economic viability of the project. The pilot phase has had the full support of the Mondelēz International Foundation, but it should become sustainable mostly through internal resources once this phase is complete. This is why the great challenge of the program involves designing, developing, and testing an on-line dissemination strategy that will allow all interested Spanish secondary schools to replicate it at a relatively low cost.

In sum, the results illustrate how each step within intervention, implementation, and utilization was linked. The PIP process was extremely useful for mapping the impact pathways, understanding realistic expectations for the program's impact, and identifying the key CCPs that must be monitored for the program processes to flow smoothly.

\section{Conclusions}

The CAL-TAS Program has been awarded the regional PAAS Prize for the best food and physical activity promotion program in schools by the Public Health Agency of the Catalan Government [16], as well as a special mention at the Nutrition, Physical Activity, and Obesity (NAOS) awards [17] under the category of Healthy Food in Schools. While the pilot intervention has shown strong evidence of its effectiveness in changing lifestyle behaviors among teenagers, the PIP analysis, presentation, and feedback received at the Granada workshop have resulted in the improvement of a number of CAL-TAS Program implementation, operations, and evaluation procedures.

The obesity epidemic is one of the greatest public health, social, and economic challenges of the 21 st century. Without a strong contribution from school-based healthy lifestyle education programs, we are not likely to reverse the epidemic. Achieving maximum impact necessitates the use of evidence-based programs that are well designed, implemented, and evaluated. The PIP methodology proved to be a powerful analysis tool that others should consider using when designing and developing school-based healthy lifestyles programs targeting teenagers.

In conclusion, CAL-TAS is an effective secondary school-based healthy lifestyle pilot program. The PIP analysis was instrumental in refining the aims of the program and in identifying CCPs to sustain and improve the quality of the program. Based on the CALTAS PIP analysis, we recommend that indicators of change in knowledge and behaviors related to diet and physical activity be used in any school-based healthy 
lifestyles program. By following these program evaluation best practices, it is likely that the impact of programs will be sustained or even improved in the future.

\section{Ethical approval of study and informed consent}

This study did not involve the collection of any primary data or access to any personal information related to the program's participants or staff and thus was exempt from the requirements regarding informed consent.

\section{References}

1. World Obesity Federation. Available at: http://www .worldobesity.org/iotf/obesity/. Accessed 5 May 2014.

2. García-Goñi M, Hernández-Quevedo C. The evolution of obesity in Spain. Eurohealth 2012;18:22-5.

3. World Health Organization. Overweight and obesity. Available at: http://www.who.int/gho/ncd/risk_factors/ overweight/en/. Accessed 5 May 2014.

4. Encuesta Nacional de Salud de España. Available at: http://www.msc.es/estadEstudios/estadisticas/encuesta Nacional/home.htm. Accessed 5 May 2014.

5. García Rada A. Study finds almost half of Spanish children are overweight or obese. BMJ 2011;343:d4218.

6. NAOS Strategy. Estrategia de nutrición, actividad física y prevención de obesidad. Available at: http://www.naos .aesan.msssi.gob.es/. Accessed 5 May 2014.

7. Kropski JA, Keckley PH, Jensen GL. School-based obesity prevention programs: an evidence-based review. Obesity 2008;16:1009-18.

8. Story M, Neumark-Sztainer D, French S. Individual and environmental influences on adolescent eating behaviors. J Am Diet Assoc 2002;102(3 suppl):S40-51.

9. Croll JK Neumark-Sztainer D, Story M. Healthy eating: what does it mean to adolescents? J Nutr Educ 2001; 33:193-8.

10. Morgan AZ, Ulrich P, Simmons KP, Gropper SS, Connell LJ, Daniels MK, Latham E, Keiley MK. Effectiveness of a multi-faceted school-based health intervention program with 4th graders in Alabama. Child Youth Serv Rev 2014:37(issue C): 46-54.

\section{Conflicts of interest and other disclosures}

This manuscript is an original work and has not been submitted for publication elsewhere. All authors reviewed and approved the article.

\section{Acknowledgments}

The program presented in this work is primarily funded by the Mondelēz International Foundation, which is the sole funder of these workshop proceedings.

11. Miller MD. Evaluation of a 2-year physical activity and healthy eating intervention in middle school children. Health Educ Res 2005;21:911-21.

12. Veugelers PJ1, Fitzgerald AL. Effectiveness of school programs in preventing childhood obesity: a multilevel comparison. Am J Public Health 2005;95:432-5.

13. Kim SS, Habicht J-P, Menon P, Stoltzfus R. How do programs work to improve child nutrition? Program impact pathways of three nongovernmental organization intervention projects in the Peruvian highlands. IFPRI Discussion Paper 01105. Washington, DC: International Food Policy Research Institute, 2011. Available at: http://www.ifpri.org/sites/default/files/publications/ ifpridp01105.pdf. Accessed 5 May 2014.

14. Guía de la Alimentación Saludable de la SENC. Sociedad Española de Nutrición Comunitaria. Available at: http://www.nutricioncomunitaria.org/generica .jsp?tipo=docu\&id=3. 14. Accessed 5 May 2014.

15. Pérez-Escamilla R, Segura-Pérez S, Damio G. Applying the Program Impact Pathways (PIP) evaluation framework to school-based healthy lifestyles programs: Workshop Evaluation Manual. Food Nutr Bull 2014;35:S97-107.

16. Programa TAS. Programa Tu i Alícia per la salud. Available at: www.programatas.com. Accessed 5 May 2014

17. Premios NAOS. 2012. Nutrición, actividad física y prevención de obesidad. Available at: http://www.naos .aesan.msssi.gob.es/naos/estrategia/premios/ganadores/ ganadores_2012.html. Accessed 5 May 2014. 